\title{
ARTICLE
}

\section{Impact of midazolam vs. saline on effect size estimates in controlled trials of ketamine as a rapid-acting antidepressant}

Samuel T. Wilkinson ${ }^{1}$, Cristan Farmer iD $^{2}$, Elizabeth D. Ballard ${ }^{2}$, Sanjay J. Mathew ${ }^{3,4}$, Michael F. Grunebaum ${ }^{5}$, James W. Murrough $^{6}$, Peter Sos ${ }^{7}$, Gang Wang ${ }^{8}$, Ralitza Gueorguieva ${ }^{1,9}$ and Carlos A. Zarate Jr. ${ }^{2}$

The goal of this study was to infer the effectiveness of midazolam as a comparator in preserving the blind in ketamine studies for mood disorders through patient-level analyses of efficacy trial outcomes. In this integrative data analysis $(k=9, N=367$ patients with mood disorders), clinical outcomes were compared across four groups: ketamine (midazolam-controlled), ketamine (salinecontrolled), midazolam, and saline. Ketamine doses ranged from 0.5 to $0.54 \mathrm{mg} / \mathrm{kg}$ and midazolam doses ranged from 0.02 to 0.045 $\mathrm{mg} / \mathrm{kg}$. The baseline-to-Day 1 effect size was $d=0.7$ (95\% Cl: $0.4-0.9)$ for ketamine (midazolam) versus midazolam and $d=1.8(95 \%$ $\mathrm{Cl}$ : 1.4-2.2) for ketamine (saline) versus saline. The effect of ketamine relative to control was larger in saline-controlled studies than in midazolam-controlled studies $(t(276)=2.32, p=0.02)$. This was driven by a comparatively larger effect under midazolam than saline $(t(111)=5.40, p<0.0001)$, whereas there was no difference between ketamine (midazolam) versus ketamine (saline) $(t(177)$ $=0.65, p=0.51$ ). Model-estimated rates of response (with $95 \% \mathrm{Cl}$ ) yielded similar results: ketamine (midazolam), $45 \%(34-56 \%)$; ketamine (saline), 46\% (34-58\%); midazolam, 18\% (6-30\%); saline, 1\% (0-11\%). The response rate for ketamine was higher than the control condition for both saline $(t(353)=7.41, p<0.0001)$ and midazolam $(t(353)=4.59, p<0.0001)$. Studies that used midazolam as a comparator yielded smaller effects of ketamine than those which used saline, which was accounted for by greater improvement following midazolam compared to saline.

Neuropsychopharmacology (2019) 44:1233-1238; https://doi.org/10.1038/s41386-019-0317-8

\section{INTRODUCTION}

Ketamine has emerged as the prototypical rapid-acting antidepressant, yet several challenges remain in both research and clinical domains. One such challenge is the integrity of the blind when ketamine is evaluated in randomized controlled trials. Due to the potent psychoactive effects of ketamine, there is a strong argument that both participants and raters may be functionally unblinded when saline is used as the comparator. In an attempt to address this problem, Murrough et al. [1] were the first to employ midazolam as an "active placebo" control condition in a ketamine trial in depression. Midazolam was selected because of similar pharmacokinetic characteristics to those of ketamine and because of its purported non-specific behavioral effects (i.e., sedation, disorientation).

Concerns persist about functional unblinding in clinical trials of standard oral antidepressants due to adverse effects [2]. This is especially so in trials involving ketamine because the acute side effects (dissociation, etc.) are pronounced. Yet most clinical trials, even those involving standard antidepressants, do not routinely assess the integrity of the blind. Because of this, it is difficult to evaluate whether midazolam is an improvement over saline with respect to maintaining the integrity of the blind. One indicator of this might be the effect size of a study, which would be expected to decrease with improved integrity of the blind. Further, studies of ketamine routinely collect scores on a measure of dissociation, which may offer some information on how closely midazolam mimics some of the distinctive behavioral side effects of ketamine. The goal of this study was to examine the effectiveness of midazolam as a comparator in preserving the blind in ketamine studies through secondary analyses of efficacy and dissociative effects.

\section{METHODS}

Data

Drawing upon previous collaborations [3], we compiled participant-level data from 9 studies $(N=367)$ where ketamine was compared either to saline or midazolam. The studies from the National Institutes of Health were conducted under a single protocol and are therefore coded as a single study [4-7]. All participants had major depressive disorder or bipolar disorder.

Patient-level data included overall depression rating scale (Montgomery-Asberg Depression Rating Scale [MADRS] or Hamilton Depression Rating Scale [HDRS]), sex, age, race, use of

\footnotetext{
${ }^{1}$ Department of Psychiatry, Yale School of Medicine, New Haven, CT, USA; ${ }^{2}$ Experimental Therapeutics and Pathophysiology Branch, National Institute of Mental Health, Bethesda, MD, USA; ${ }^{3}$ Menninger Department of Psychiatry \& Behavioral Sciences, Baylor College of Medicine, Houston, USA; ${ }^{4}$ Mental Health Care Line, Michael E. Debakey VA Medical Center, Houston, USA; ${ }^{5}$ Department of Psychiatry, Columbia University, Medical Center and New York State Psychiatric Institute, New York, USA; ${ }^{6}$ Department of Psychiatry, Icahn School of Medicine at Mount Sinai, New York, USA; ${ }^{7}$ Department of Psychiatry, First Faculty of Medicine, Charles University in Prague, Prague, Czech Republic; ${ }^{8}$ Beijing Anding Hospital, Capital University of Medical Sciences, Beijing, China and ${ }^{9}$ Department of Biostatistics, Yale School of Public Health, New Haven, CT, USA

Correspondence: Samuel T. Wilkinson (samuel.wilkinson@yale.edu)

These authors contributed equally: Samuel T. Wilkinson, Cristan Farmer
}

Received: 27 November 2018 Accepted: 8 January 2019

Published online: 17 January 2019 
concomitant medications, and inpatient or outpatient status at time of infusion. The 17-item HDRS was converted to MADRS as previously described [8] for five studies [5, 9-12] wherein the MADRS was not collected. Data collection points varied across studies, so the primary outcome was limited to baseline and Day 1 post-infusion, which were available for all studies. The Clinician Administered Dissociative State Scale (CADSS) was administered 40-60 min after start of infusion in most studies [1, 4-7, 9-13]. For comparability to the parallel-arm trials, data from only the first period of crossover studies were included in this analysis $(k=4$, $n=151)$.

\section{Statistical analysis}

Patients were categorized into one of four groups, based on the condition to which they were assigned: ketamine (midazolam), used in studies with midazolam as a comparator; ketamine (saline), used in studies with saline as a comparator; midazolam; and saline. We compared the change in MADRS at Day 1 postinfusion, using a linear mixed model with a random effect of study. The residuals from each timepoint were allowed to covary within subject (unstructured matrix, estimated separately by treatment condition). Fixed effects of treatment, time, and their interaction were included, with Satterthwaite correction to the denominator degrees of freedom. The differences between treatment groups were evaluated with a series of between-group contrasts of change from baseline to Day 1 . There was little variability in baseline CADSS scores, which clustered at zero, so the effect of treatment on CADSS was evaluated using only the 30-40-min timepoint. To conform to distributional assumptions, CADSS scores were natural-log transformed (after adding a constant of 1 ). This was an intentto-treat analysis and individuals with missing data $(n=2$, both Day 1) were not excluded. Cohen's d was calculated using the least-square mean estimated differences, standard errors, and DF of a given test. All analyses were performed using SAS/STAT Version 9.3 .

\section{RESULTS}

Demographic and clinical characteristics

We obtained participant-level data from $k=9$ studies $(N=367$ subjects with mood disorders; $n=106$ participants in Ketamine (midazolam), $n=81$ in Ketamine (saline), $n=83$ in Midazolam, and $n=97$ in Saline) (Table 1). The average age of the pooled sample was 42.2 years old (SD 12.5), and $56.7 \%$ were female. The majority of participants were diagnosed with Major Depressive Disorder (82.6\%) (versus Bipolar Disorder).

\section{Change in MADRS total score}

Baseline differences in MADRS score across the treatment groups were non-significant (all $p>0.22$ ). Significant improvement from baseline to Day 1 was observed in all conditions (Fig. 1, panel a), although the degree of improvement varied across conditions (Fig. 1, panel b). The improvement observed in ketamine (midazolam) $($ model-estimated mean improvement $=13.6, \mathrm{SE}=$ 1.1 ) exceeded that in midazolam (mean $=7.0, \mathrm{SE}=0.9)$ (comparison: $t(185)=19.94, p<0.0001)$. The effect size was $d=0.7(95 \%$ $\mathrm{Cl}$ : 0.4-0.9). Similarly, the improvement observed in ketamine (saline) (mean $=12.5, \mathrm{SE}=1.2$ ) exceeded that of saline (mean $=$ 1.6, SE $=0.4$ ) (comparison: $t(96.5)=80.8, p<0.0001$ ). The effect size was $d=1.8(95 \% \mathrm{Cl}$ : 1.4-2.2). The effect of ketamine relative to control was larger in saline-controlled studies than in midazolam-controlled studies $(t(276)=2.32, p=0.02)$. This was driven by a comparatively larger effect under midazolam than saline $(t(111)=5.40, p<0.0001)$, whereas there was no difference between ketamine (midazolam) versus ketamine (saline) $(t(177)=$ $0.65, p=0.51)$.
Dichotomous outcomes: response at Day 1

We also examined dichotomous outcomes of Responder (improvement of at least $50 \%$ at Day 1) and Non-Responder (worsening, or improvement of less than $50 \%$ at Day 1) categories. Given this binary outcome, a generalized linear mixed model was used. Model-estimated rates of response (with 95\% Cl) in each group were as follows: ketamine (midazolam), 45\% (34-56\%); ketamine (saline), 46\% (34-58\%); midazolam, 18\% (6-30\%); saline, $1 \%(0 \%-11 \%)$ (Fig. 1, Panel c). The response rate for ketamine was higher than the control condition for both saline $(t(353)=7.41, p$ $<0.0001)$ and midazolam $(t(353)=4.59, p<0.0001)$. There was a greater difference between ketamine and saline than between ketamine and midazolam in proportion of responders $(t(353)=$ $2.15, p=0.03$ ). This represents a number-needed-to-treat (NNT) of 2.2 for ketamine in saline-controlled studies and 3.7 for ketamine in midazolam-controlled studies.

\section{Participant blinding}

Three midazolam-controlled studies $[1,10,11]$ directly assessed the integrity of the blind by asking participants to guess to which group they were assigned. For comparability, we selected guess data from the 24-h timepoint from each study, and coded responses of "I don't know" or "not sure" as incorrect. We tested whether the rate of correct responses was different from $50 \%$ (the level of chance with two possible outcomes, correct or incorrect). The rate of correct guess in neither the Murrough et al. [1] study nor the Grunebaum et al. [11] study differed from chance $(p=0.81$ and $p=0.37$, respectively). The rate of correct guess in the smaller $(N=16)$ Grunebaum et al. [10] study was higher and nominally different from chance $(75 \%, p=0.046)$.

\section{Dissociative effects}

Data from the CADSS were available for 286 participants in seven studies (see Table 1). Both control conditions produced relatively little dissociation as measured by CADSS total score (Saline, $M=$ $0.42, \mathrm{SE}=0.15$; Midazolam, $M=0.65, \mathrm{SE}=0.13$, log-transformed scores); these scores did not differ from one another $(t(277)=1.14$, $p=0.25)$. Notably, CADSS scores were higher in the Ketamine (saline) condition $(M=3.01, \mathrm{SE}=0.19)$ than in Ketamine (midazolam) $(M=2.27, \mathrm{SE}=0.15$, log-transformed scores) (comparison, $t(277)=3.04, p=0.003)$. The effect of ketamine relative to control was larger in the saline-controlled studies than in the midazolam-controlled studies $(t(277)=4.28, p<0.0001)$, though this was largely driven by differences between ketamine (Midazolam) and ketamine (Saline) groups.

\section{DISCUSSION}

The goal of this study was to examine the effect of midazolam vs. saline on drug-comparator effect size as a proxy for preserving the blind in ketamine studies through analysis of efficacy and dissociative effects from previously published clinical trials. We found that the average antidepressant effect of ketamine was smaller when compared with midazolam than when compared with saline using both continuous (depression rating scales) and categorical outcomes (response rates). The difference in effect size was driven by greater improvement in the midazolam group compared to the saline group. One interpretation of the smaller effect size is that midazolam was superior to saline in preserving the integrity of the blind. While we were not able to assess this directly, we did find that neither saline nor midazolam produced appreciable dissociative side effects as measured by CADSS and there was no difference between these controls groups in this respect. Still, the available data indicated that patients were unable to distinguish between midazolam and ketamine. However, alternative explanations for the difference in effect size depending on comparator, such as the hypothesis that a single 


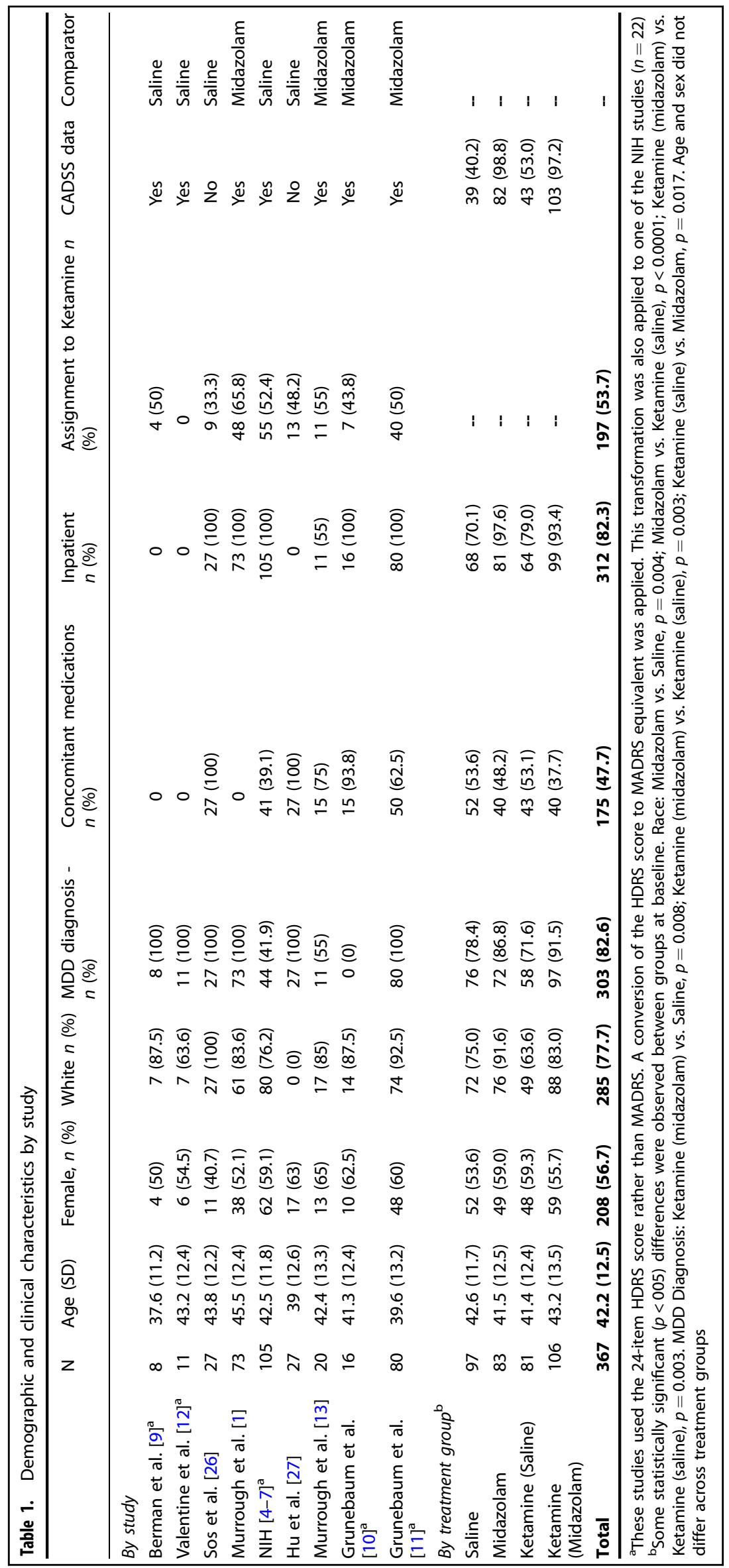



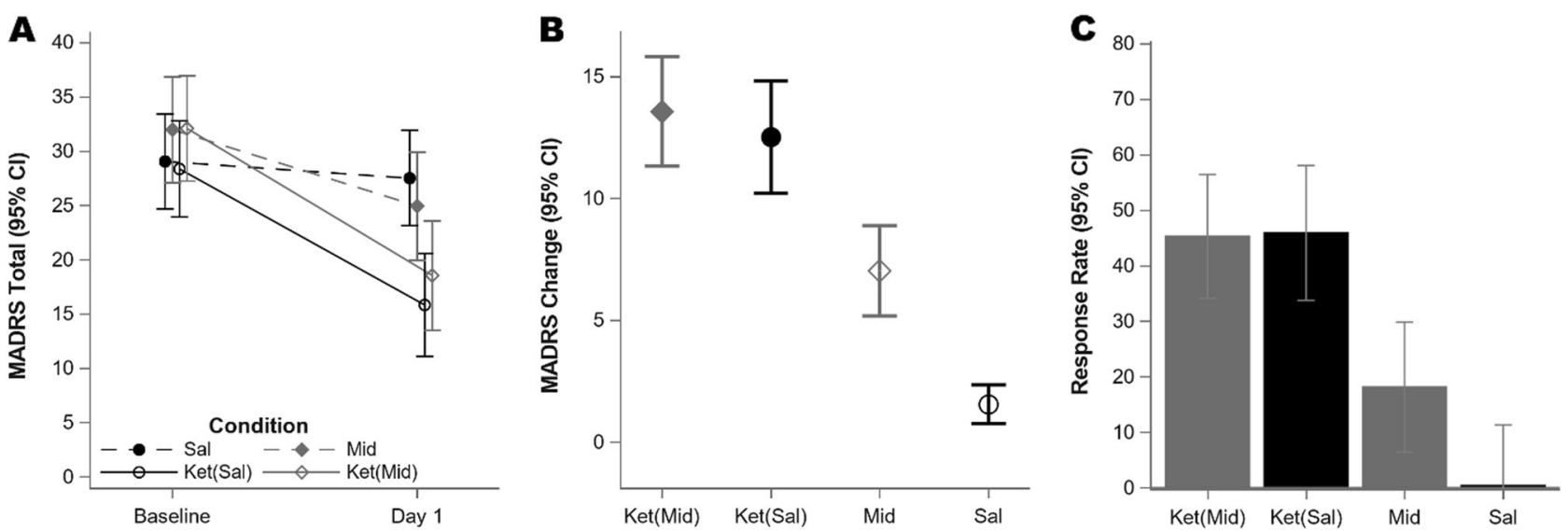

Fig. 1 Results of mixed models evaluating change in MADRS score by treatment ( $95 \%$ confidence intervals). Note: Ket(Mid) $=$ Ketamine (in midazolam-controlled studies, $N=106) ; \mathrm{Ket}(\mathrm{Sal})=$ Ketamine (in saline-controlled studies, $N=81)$; Mid $=$ Midazolam $(N=83) ; \mathrm{Sal}=\mathrm{Saline}(N=$ 97). Plotted values are model-estimated means from mixed models predicting MADRS (panel a and $\mathbf{b}$ ) and predicted binary response ( $\geq 50 \%$ improvement in MADRS vs. $<50 \%$ improvement) from treatment at $24 \mathrm{~h}$

infusion of midazolam has antidepressant effects that extend for $24 \mathrm{~h}$, cannot be excluded. A three-arm study comparing ketamine, midazolam, and saline would be necessary to definitively answer this question.

Blinding is of central importance in clinical trials. In concert with randomization and allocation concealment, blinding reduces bias and ensures clinical investigators that drug-comparator differences represent effects directly attributable to the intervention of interest, and not regression to the mean, the natural variation in the course of the illness, expectancy or other non-specific or placebo effects. As noted by Murrough and colleagues [1], there is likely no perfect control condition for ketamine. While midazolam shares some pharmacological properties with ketamine (fast onset of action, short-half life, available by infusion), the acute side effects profile of ketamine is rather unique among readily available pharmacological agents. These acute effects of ketamine are often difficult for patients to describe, but are sometimes referred to as "dissociative" [14] or even "mystical" [15]. Our findings show that midazolam may narrow the drug-comparator difference in acute antidepressant effects. We also found that in two midazolam-controlled studies of respectable sample size $(N=$ $80,73)$, patients were unable to correctly guess whether they had received the medication $[1,11]$. Notably, in a small pilot study ( $N$ $=16$ ) where midazolam was used, patients guessed better than chance [10]. However, we found no evidence that a possible improvement in blinding was due to midazolam inducing dissociative effects (as measured by the CADSS). Furthermore, as other investigators have noted, ketamine is readily distinguished from lorazepam, another benzodiazepine, using a scale designed to assess mystical side effects [15].

Several limitations of the current study require comment. It is important to emphasize that none of the trials included in our analysis compared midazolam directly with saline. Hence, while it is reassuring that there were no baseline differences in depression severity, we cannot be assured that all groups are comparable in other unmeasured potential confounding variables. It is also not possible to control for methodology-related confounds, such as differences in study design or population. Owing to the differences in anxiety sub-items of the different scales used in this study, it was not possible to examine whether the smaller drug-comparator difference seen in midazolam-controlled studies could be explained entirely by the expected anti-anxiety effects of midazolam. Notably, this limitation is mitigated by the fact that the primary outcome in this study was assessed 1 day following drug exposure. Given the short half-life of midazolam $(\sim 2 \mathrm{~h})$ and the fact that benzodiazepines do not generally have anxiolytic effects that endure beyond the time the drug is in the body, it is unlikely that the anxiolytic effects of the drug explain the differences in effect size between midazolam-controlled and saline-controlled studies. At the very least, a single dose of benzodiazepine has not been shown to have rapid-acting antidepressant effects earlier than one week, although continuous exposure to benzodiazepine can produce antidepressant effects that are detectable as early as one week [16]. Another limitation is that these studies only evaluated a narrow dose range of ketamine $(0.5-0.54 \mathrm{mg} / \mathrm{kg})$ and did not consider other doses. A recent dosefinding study suggests that this is the optimal antidepressant dose [17]. However, lower doses of ketamine $(0.1-0.2 \mathrm{mg} / \mathrm{kg})$ produce less dissociative effects and hence the comparability of midazolam and saline as control conditions against low-dose ketamine is unknown. Additionally, in order to combine data across studies, we used converted MADRS scores in some cases. This limitation is mitigated by consistent findings using the dichotomous outcome of response ( $\geq 50 \%$ improvement), where outcomes from different studies can be directly compared without scale conversion. Finally, only three studies, all with midazolam as a comparator, formally assess the integrity of the blind by asking participants to guess their treatment assignment. While we were unable to compare midazolam to saline, it is noteworthy that in the two largest midazolam-controlled studies $(N=73, N=80)$, patients were unable to guess correctly their treatment assignment at a rate higher than expected by chance. It should be noted that these studies used different doses of midazolam [1, 11].

Despite these limitations, our findings suggest that midazolam may improve the integrity of the blind in ketamine clinical trials when used in lieu of saline. As noted, even if midazolam has antidepressant or anxiolytic effects, benzodiazepines have not been shown to have enduring psychotropic effects that last beyond the period that the drug is in the body. The short half-life of midazolam $(\sim 2 \mathrm{~h})$ makes it unlikely that significant drug effects would be detected the day following infusion.

Furthermore, the extremely low placebo response rate observed in the saline group in our study (1\%) is less than is generally found in clinical trials of antidepressants, even with a population with some level of treatment resistance. For example, several clinical trials of lanicemine, which is also given intravenously but cannot readily be distinguished from saline by acute effects, tested in protocols of varying lengths found varying placebo response rates of 15-31\% [18-20]. The "placebo" response rate of the midazolam group (18\%) in our study is more consistent with placebo response rates of these and other trials of antidepressants [21, $22]$, though most antidepressant trials assess response rates over a 
much longer period (4-6 weeks) as opposed to $24 \mathrm{~h}$. It should be noted that a recent midazolam-controlled study examining four different doses of ketamine showed the "placebo" response rate in the midazolam group continued to increase from $11 \%$ at $24 \mathrm{~h}$ to $33 \%$ at $72 \mathrm{~h}$ post-infusion [17]; because there was no saline group, it is uncertain whether this effect was due to the midazolam or to non-specific "placebo" effects. This last point underscores that the expected placebo response in trials of rapid antidepressants is not well established. The expectation of patients to potentially feel better within hours or days following a single drug exposure may produce different placebo response rates than those seen in clinical trials of standard antidepressants.

It is important to note that even if midazolam is superior to saline as a comparator for clinical trials involving ketamine for blinding purposes, other factors may be considered depending on the setting and the goal of the study; hence, midazolam may not be the best choice for all purposes. For example, midazolam could confound clinical studies where biomarkers are used as outcomes. Another consideration is safety, as it may not be ethical or practical in large clinical trials to expose participants in the comparator group to repeated doses of midazolam. Midazolam can cause respiratory depression and lower blood pressure, and may require a higher level of medical supervision. While blinding is an important factor in clinical trials, these other aspects may prompt consideration of alternative comparators. Notably, several FDA-registered trials of compounds with significant dissociative effects (esketamine, ketamine/NRX-100) are using saline as the comparator condition [23, 24] (clinical trial identifiers: NCT02417064, NCT02422186, NCT03395392, NCT03396601).

In sum, our study found a smaller antidepressant effect size in single-infusion ketamine studies when midazolam was used as the comparator than when saline was used as the comparator. This finding was driven by greater improvement in the midazolam group compared to the saline group - to a degree more in line with other antidepressant trials than saline-and suggests that a midazolam comparator may yield a more realistic estimate of ketamine's antidepressant effect. While our results suggest that midazolam may improve the integrity of the blind, alternative explanations, such as the hypothesis that midazolam has enduring antidepressant effects [25], cannot be excluded. Even if midazolam is superior compared to saline in preserving the blind in ketamine studies, other trial design factors, such as inclusion of biomarkers, safety, and overall trial feasibility, may warrant consideration of the choice of another comparator.

\section{ACKNOWLEDGEMENTS}

The content is solely the responsibility of the authors and does not necessarily represent the official views of the Agency for Healthcare Research and Quality. We also acknowledge support from the American Foundation for Suicide Prevention, Brain and Behavior Research Foundation (formerly NARSAD), the Patient-Centered Outcomes Research Institute, and the Robert E. Leet and Clara Guthrie Patterson Foundation (STW).

\section{FUNDING AND DISCLOSURE}

This work was supported in part by grant number K12HS023000 from the Agency for Healthcare Research and Quality (STW). STW receives or has received in the last 36 months funding from Janssen Pharmaceuticals. He has consulted for Janssen Pharmaceuticals. CAZ is a full-time U.S government employee. He is listed as a coinventor on a patent for the use of ketamine in major depression and suicidal ideation. CAZ is listed as a co-inventor on a patent for the use of $(2 R, 6 R)$ hydroxynorketamine, (S)-dehydronorketamine and other stereoisomeric dehydro and hydroxylated metabolites of (R,S)-ketamine metabolites in the treatment of depression and neuropathic pain. CAZ is listed as co-inventor on a patent application for the use of $(2 R, 6 R)$-hydroxynorketamine and $(2 S, 6 S)$-hydroxynorketamine in the treatment of depression, anxiety, anhedonia, suicidal ideation, and post-traumatic stress disorders. CAZ has assigned his patent rights to the U.S. government but will share a percentage of any royalties that may be received by the government. SJM is supported through the use of facilities and resources at the Michael E. Debakey VA
Medical Center; he is a consultant for: Allergan, Alkermes, Bracket, Clexio, Fortress Biotech, Sage Therapeutics research support: Janssen, NeuroRx, Vistagen, Department of Veterans Affairs, NIMH, PCORI. RG discloses consulting fees for Palo Alto Health Sciences, Knopp Biosciences and Mathematica Policy Research, royalties from book "Statistical Methods in Psychiatry and Related Fields" published by CRC Press, and a provisional patent submission by Yale University: Chekroud, AM., Gueorguieva, R., \& Krystal, JH. "Treatment Selection for Major Depressive Disorder" [filing date 3rd June 2016, USPTO docket number Y0087.70116US00]. In the past 3 years, Dr. Murrough has provided consultation services to Boehreinger Ingelheim, Sage Therapeutics, Novartis, Allergan, Fortress Biotech, Janssen Research and Development, Medavante-Prophase, and Global Medical Education (GME) and has received research support from Avanir Pharmaceuticals, Inc. JWM is named on a patent pending for neuropeptide $Y$ as a treatment for mood and anxiety disorders. The Icahn School of Medicine (employer of JWM) is named on a patent and has entered into a licensing agreement and will receive payments related to the use of ketamine if it is approved for the treatment of depression. JMW is not named on this patent and will not receive any payments. CF, MFG, EB, GW, and PS report no disclosures.

\section{ADDITIONAL INFORMATION}

Publisher's note: Springer Nature remains neutral with regard to jurisdictional claims in published maps and institutional affiliations.

\section{REFERENCES}

1. Murrough JW, losifescu DV, Chang LC, Al Jurdi RK, Green CE, Perez AM, et al. Antidepressant efficacy of ketamine in treatment-resistant major depression: a two-site randomized controlled trial. Am J Psychiatry. 2013;170:1134-42.

2. Perlis RH, Ostacher M, Fava M, Nierenberg AA, Sachs GS, Rosenbaum JF. Assuring that double-blind is blind. Am J Psychiatry. 2010;167:250-2.

3. Wilkinson ST, Ballard ED, Bloch MH, Mathew SJ, Murrough JW, Feder A, et al. The effect of a single dose of intravenous ketamine on suicidal ideation: a systematic review and individual participant data meta-analysis. Am J Psychiatry. 2018;175:150-8.

4. Nugent AC, Ballard ED, Gould TD, Park LT, Moaddel R, Brutsche NE, et al. Ketamine has distinct electrophysiological and behavioral effects in depressed and healthy subjects. Mol Psychiatry. 2018;8:1.

5. Zarate CA Jr., Singh JB, Carlson PJ, Brutsche NE, Ameli R, Luckenbaugh DA, et al. A randomized trial of an $\mathrm{N}$-methyl-D-aspartate antagonist in treatment-resistant major depression. Arch Gen Psychiatry. 2006;63:856-64.

6. Zarate CA Jr, Brutsche NE, Ibrahim L, Franco-Chaves J, Diazgranados N, Cravchik A, et al. Replication of ketamine's antidepressant efficacy in bipolar depression: a randomized controlled add-on trial. Biol Psychiatry. 2012;71:939-46.

7. Diazgranados N, Ibrahim L, Brutsche NE, Newberg A, Kronstein P, Khalife S, et al. A randomized add-on trial of an $\mathrm{N}$-methyl-D-aspartate antagonist in treatmentresistant bipolar depression. Arch Gen Psychiatry. 2010;67:793-802.

8. Carmody TJ, Rush AJ, Bernstein I, Warden D, Brannan S, Burnham D, et al. The Montgomery Asberg and the Hamilton ratings of depression: a comparison of measures. Eur Neuropsychopharmacol. 2006;16:601-11.

9. Berman RM, Cappiello A, Anand A, Oren DA, Heninger GR, Charney DS, et al. Antidepressant effects of ketamine in depressed patients. Biol Psychiatry. 2000;47:351-4.

10. Grunebaum MF, Ellis SP, Keilp JG, Moitra VK, Cooper TB, Marver JE, et al. Ketamine versus midazolam in bipolar depression with suicidal thoughts: a pilot midazolam-controlled randomized clinical trial. Bipolar Disord. 2017;19: 176-83.

11. Grunebaum MF, Galfalvy HC, Choo TH, Keilp JG, Moitra VK, Parris MS, et al. Ketamine for rapid reduction of suicidal thoughts in major depression: a midazolam-controlled randomized clinical trial. Am J Psychiatry. 2018;175: 327-35.

12. Valentine GW, Mason GF, Gomez R, Fasula M, Watzl J, Pittman B, et al. The antidepressant effect of ketamine is not associated with changes in occipital amino acid neurotransmitter content as measured by [(1)H]-MRS. Psychiatry Res. 2011;191:122-7.

13. Murrough JW, Soleimani L, DeWilde KE, Collins KA, Lapidus KA, lacoviello BM, et al. Ketamine for rapid reduction of suicidal ideation: a randomized controlled trial. Psychol Med. 2015;45:3571-80.

14. Domino EF, Chodoff P, Corssen G. Pharmacologic effects of $\mathrm{Cl}-581$, a new dissociative anesthetic, in man. Clin Pharmacol Ther. 1965;6:279-91.

15. Dakwar E, Anerella C, Hart CL, Levin FR, Mathew SJ, Nunes EV. Therapeutic infusions of ketamine: do the psychoactive effects matter? Drug Alcohol Depend. 2014;136:153-7. 
Impact of midazolam vs. saline on effect size estimates in controlled... ST Wilkinson et al.

16. Rickels K, Feighner JP, Smith WT. Alprazolam, amitriptyline, doxepin, and placebo in the treatment of depression. Arch Gen Psychiatry. 1985;42:134-41.

17. Fava M, Freeman MP, Flynn M, Judge H, Hoeppner BB, Cusin C, et al. Doubleblind, placebo-controlled, dose-ranging trial of intravenous ketamine as adjunctive therapy in treatment-resistant depression (TRD). Mol Psychiatry. 2018; https://doi.org/10.1038/s41380-018-0256-5. [Epub ahead of print].

18. Sanacora G, Johnson MR, Khan A, Atkinson SD, Riesenberg RR, Schronen JP, et al. Adjunctive Lanicemine (AZD6765) in patients with major depressive disorder and history of inadequate response to antidepressants: a randomized, placebocontrolled study. Neuropsychopharmacology. 2017;42:844-53.

19. Sanacora G, Smith MA, Pathak S, Su HL, Boeijinga PH, McCarthy DJ, et al. Lanicemine: a low-trapping NMDA channel blocker produces sustained antidepressant efficacy with minimal psychotomimetic adverse effects. Mol Psychiatry. 2014;19:978-85.

20. Zarate CA Jr., Mathews D, Ibrahim L, Chaves JF, Marquardt C, Ukoh I, et al. A randomized trial of a low-trapping nonselective $\mathrm{N}$-methyl-D-aspartate channel blocker in major depression. Biol Psychiatry. 2013;74:257-64.

21. Walsh BT, Seidman SN, Sysko R, Gould M. Placebo response in studies of major depression: variable, substantial, and growing. JAMA. 2002;287:1840-7.
22. Dunlop BW, Thase ME, Wun CC, Fayyad R, Guico-Pabia CJ, Musgnung J, et al. A metaanalysis of factors impacting detection of antidepressant efficacy in clinical trials: the importance of academic sites. Neuropsychopharmacology. 2012;37:2830-6.

23. Canuso CM, Singh JB, Fedgchin M, Alphs L, Lane R, Lim P, et al. Efficacy and safety of intranasal esketamine for the rapid reduction of symptoms of depression and suicidality in patients at imminent risk for suicide: results of a double-blind, randomized, placebo-controlled study. Am J Psychiatry. 2018;7:620-30.

24. Daly EJ, Singh JB, Fedgchin M, Cooper K, Lim P, Shelton RC, et al. Efficacy and safety of intranasal esketamine adjunctive to oral antidepressant therapy in treatment-resistant depression: a randomized clinical trial. JAMA Psychiatry. 2018;75:139-48.

25. Petty F, Trivedi MH, Fulton $M$, Rush AJ. Benzodiazepines as antidepressants: does GABA play a role in depression? Biol Psychiatry. 1995;38:578-91.

26. Sos P, Klirova M, Novak T, Kohutova B, Horacek J, Palenicek T. Relationship of ketamine's antidepressant and psychotomimetic effects in unipolar depression. Neuro Endocrinol Lett. 2013;34:287-93.

27. Hu YD, Xiang YT, Fang JX, Zu S, Sha S, Shi H, et al. Single i.v. ketamine augmentation of newly initiated escitalopram for major depression: results from a randomized, placebo-controlled 4-week study. Psychol Med. 2016;46:623-35. 